\title{
MCM2 and TIP30 are prognostic markers in squamous cell/adenosquamous carcinoma and adenocarcinoma of the gallbladder
}

\author{
ZIRU LIU ${ }^{1}$, ZHULIN YANG $^{2}$, SONG JIANG ${ }^{2}$, QIONG ZOU ${ }^{3}$, YUAN YUAN ${ }^{3}$, JINGHE LI $^{4}$, \\ DAIQIANG $\mathrm{LI}^{5}$, LUFENG LIANG ${ }^{6}$, MEIGUI CHEN ${ }^{7}$ and SENLIN CHEN ${ }^{8}$
}

${ }^{1}$ Department of Minimal Invasive Surgery; ${ }^{2}$ Research Laboratory of Hepatobiliary Diseases, The Second Xiangya Hospital,
Central South University, Changsha, Hunan $410011 ;{ }^{3}$ Department of Pathology, The Third Xiangya Hospital,
Central South University, Changsha, Hunan $410013 ;{ }^{4}$ Department of Pathology, Basic School of Medicine,
Changsha, Hunan $410078 ;{ }^{5}$ Department of Pathology, The Second Xiangya Hospital, Central South University,
Changsha, Hunan $410011 ;{ }^{6}$ Department of Hepatobiliary and Pancreatic Surgery, Hunan Provincial People's Hospital,
Changsha, Hunan $410007 ;{ }^{7}$ Department of Pathology, Loudi Central Hospital, Loudi, Hunan 417011;
${ }^{8}$ Department of Pathology, Hunan Provincial Tumor Hospital, Changsha, Hunan 410013, P.R. China

Received September 23, 2015; Accepted September 30, 2016

DOI: $10.3892 / \mathrm{mmr} .2016 .5851$

\begin{abstract}
The clinicopathological and biological characteristics of squamous cell/adenosquamous carcinoma (SC/ASC) of the gallbladder remain to be fully elucidated, due to the fact that it is a rare gallbladder cancer subtype. In the current study, the expression of minichromosome maintenance complex component 2 (MCM2) and HIV-1 tat interactive protein 2 (TIP30) was measured in 46 cases of SC/ASC and 80 adenocarcinomas (AC) using immunohistochemistry. Positive MCM2 and negative TIP30 expression were significantly associated with large tumor size, high TNM stage, invasion, lymph node metastasis and lack of surgical curability in SC/ASC and AC. Positive MCM2 and negative TIP30 expression were significantly associated with poor differentiation in AC, whereas only MCM2 was correlated with differentiation in SC/ASC. Univariate Kaplan-Meier analysis demonstrated that positive MCM2 and negative TIP30 expression, the degree of differentiation, tumor size, TNM stage, invasion, lymph node metastasis and surgical curability were significantly associated with post-operative survival in patients with SC/ASC and AC. Multivariate Cox regression analysis demonstrated that positive MCM2 and negative TIP30 expression, the degree of differentiation, tumor size, TNM stage,
\end{abstract}

Correspondence to: Dr Zhulin Yang, Research Laboratory of Hepatobiliary Diseases, The Second Xiangya Hospital, Central South University, 139 Renmin Road, Changsha, Hunan 410011, P.R. China

E-mail: yangzhulin8@sina.com

Key words: MCM2, TIP30, gallbladder neoplasms, prognosis, neoplasm metastasis invasion, lymph node metastasis and lack of surgical curability were also independent predictors of poor prognosis in patients with SC/ASC and AC. These data suggest that positive MCM2 and negative TIP30 expression are closely correlated with the clinical, pathological and biological parameters, in addition to poor prognosis in patients with gallbladder cancer.

\section{Introduction}

Gallbladder cancers (GBCs) are the fifth most common type of gastrointestinal cancer and the most common biliary tract malignancy in the USA $(1,2)$. The prognosis of GBC is poor with a high mortality. Early diagnosis is rare due to the lack of specific signs or symptoms (3). Consequently, over $90 \%$ of patients with GBC are diagnosed at an inoperable stage with significant invasion and metastasis to other organs (4). The majority of GBCs are adenocarcinomas (AC; >90\%) (5), whereas other histopathological subtypes including mucinous, papillary, and squamous subtypes are observed only rarely (2). Squamous cell/adenosquamous carcinoma (SC/ASC) represents $1-12 \%$ of GBCs $(2,6)$. The clinicopathological characteristics of SC/ASC remain to be well documented, due to the fact that the majority of currently available data describe individual case reports or the analysis of small case series. Therefore, therapeutic interventions for SC/ASC remain to be fully elucidated (7). Although biomarkers for predicting the prognosis of $\mathrm{AC}$ have been investigated, none of the proposed markers have yet reached clinical application (8). Notably, no biomarkers associated with the progression and prognosis of SC/ASC have been reported. Therefore, it is important to document the clinicopathological and biological characteristics of SC/ASC.

$\mathrm{Ki}-67$ and proliferating cell nuclear antigen (PCNA) are two conventional proliferation markers used for routine clinical diagnosis and/or prognostic prediction for several types of cancer (9). However, these two markers are associated 
with significant problems. For example, Ki-67 serves a role in ribosome biosynthesis, and is not directly responsible for cell proliferation (10). In addition, the labeling index of Ki-67 immunostaining is usually affected by external factors such as nutrient deprivation (11). Notably, the precise function of Ki-67 remains unclear $(12,13)$. PCNA is a marker of DNA replication that is also required for DNA repair. Therefore, it is a less specific marker $(9,14)$. Previously, geminin was identified as a novel tumor cell proliferation marker. However, geminin expression was restricted to $S, G_{2}$ and early $M$ phase of the cell cycle $(9,14)$, thus the labeling index of geminin identifies only the number of actively proliferating cells that have passed $\mathrm{G}_{1}$ phase. Therefore, there remains a requirement for the identification of more specific and precise markers to facilitate patient diagnosis and/or prognosis.

The minichromosome maintenance (MCM) complex components 2-7 are essential for DNA replication in all eukaryotic cells, and are expressed more frequently in cancer cells compared with normal cells (15). Unlike Ki-67 and PCNA, MCM proteins are required in cycling cells, however are absent from quiescent cells. Immunostaining with antibodies against MCM identified a greater number of cells in the cell cycle than that of either PCNA or Ki-67 (16). This supports the potential clinical applications of MCM proteins as specific markers of proliferation. In addition, MCM proteins have demonstrated potential for diagnostic and prognostic applications in several types of human malignancies $(9,14)$. For example, multivariate analysis identified MCM expression as an independent negative predictor of patient survival (9). in addition, MCM expression could be expanded to population screening for neoplasia at a range of organ sites at which cells could be obtained for cytological analysis (17-19). However, the expression of MCM in GBC remains to be assessed.

HIV-1 tat interactive protein 2 (TIP30) was identified as a transcriptional cofactor that suppresses metastasis by inhibiting tumor growth (20) and angiogenesis $(21,22)$, and induces apoptosis (23). Li et al (24) demonstrated that weak or negative expression of TIP30 contributed to the development and progression of gastric cancer. When TIP30 expression was silenced, hepatocellular carcinoma and other tumors developed spontaneously in mice (25). In contrast, the ectopic expression of TIP30 increased the expression of a subset of pro-apoptotic genes (23), and subsequently inhibited tumorigenesis in liver and lung cells. The direct involvement of MCM in DNA replication and TIP30 in cell proliferation and apoptosis suggests that these molecules are potential targets for gene therapy. Therefore, the identification of proliferation-specific markers, including MCM and TIP30 expression, is important for the development of novel GBC treatments.

In the current study, the expression of MCM2 and TIP30 in surgically resected specimens, including AC and SC/ASC, was examined using immunohistochemistry. The correlation between MCM2 and TIP30 expression and the clinicopathological characteristics and prognosis of AC and SC/ASC were evaluated and compared.

\section{Materials and methods}

Case selection. The current study was pre-approved by The Ethics Committee for Human Research, Central South
University (Changsha). A total of 46 SC/ASC samples that underwent surgical resection or biopsy were diagnosed from a total of 1,060 GBC samples collected between January 1995 and December 2009 from 7 hospitals. A total of $80 \mathrm{AC}$ samples with available survival information were selected randomly from 1060 GBC samples for comparison in the present study. Among the 46 patients with SC/ASC, 27 were female and 19 were male $(\mathrm{F} / \mathrm{M}=1.42)$ aged $35-82$ (mean, $55.8 \pm 9.6)$ years. Among the 80 patients with AC, 54 were female and 26 were male (F/M=2.08), aged 33-80 (mean, 53.8 \pm 9.9 ) years. Surgery included radical resection for 14 SCs/ASCs and 26 ACs, palliative surgery for $18 \mathrm{SCs} / \mathrm{ASCs}$ and $28 \mathrm{ACs}$, and no operation for 14 SCs/ASCs and 26 ACs with biopsy alone. Survival information of all $46 \mathrm{SC} / \mathrm{ASC}$ and $80 \mathrm{AC}$ patients was obtained through letters and phone calls. The follow-up time of the study was 2 years. Individuals whom survived longer than 2 years were included in the analysis as censored cases.

Immunohistochemistry staining. Mouse anti-MCM2 (sc-373702), mouse anti-TIP30 (sc-55343) and horseradish peroxidase (HRP)-conjugated anti-mouse secondary antibodies (sc-2010) were purchased from Santa Cruz Biotechnology, Inc. (Santa Cruz, CA, USA). Staining was performed using the peroxidase-based EnVision ${ }^{\mathrm{TM}}$ Detection kit (Dako North America, Inc., Carpinteria, CA, USA) following the manufacturer's instructions. Briefly, $4 \mu \mathrm{M}$ sections were cut from routinely paraffin-embedded $\mathrm{AC}$ and SC/ASC tissues. The sections were deparaffinized and incubated with $3 \% \mathrm{H}_{2} \mathrm{O}_{2}$ for $15 \mathrm{~min}$, and soaked with phosphate-buffered saline (PBS) for $3 \times 5 \mathrm{~min}$. They were then incubated with mouse anti-MCM2 (1:100 dilution) or mouse anti-TIP30 (1:100) antibodies for $1 \mathrm{~h}$ at room temperature. Subsequent to rinsing the sections with PBS three times, solution A (containing the HRP-conjugated secondary antibody) was added and incubated for $30 \mathrm{~min}$. DAB substrate was then added, followed by hematoxylin counter-staining. The slides were subsequently dehydrated and soaked in xylene for $3 \times 5 \mathrm{~min}$. Positive sections purchased from Wuhan Boster Biological Technology, Ltd. (Wuhan, China) were used as the positive control, whereas replacing the primary antibody with 5\% fetal bovine serum was used as the negative control. The percentage of positive cells was calculated from 500 cells in 10 random fields. Cases with $\geq 25 \%$ positive cells were considered to be positive, whereas samples with $<25 \%$ positive cells were negative.

Statistical analysis. Data were analyzed using SPSS software, version 14.0 (SPSS, Inc., Chicago, IL, USA). The associations between MCM2 and TIP30 expression with histological or clinical factors were analyzed using $\chi^{2}$ or Fisher's exact test. Kaplan-Meier and time series analyses were used for univariate survival analysis. Cox proportional hazards model was used for multivariate analysis, and to determine the $95 \%$ confidence interval. $\mathrm{P}<0.05$ was considered to indicate a statistically significant difference.

\section{Results}

Comparison of clinicopathological characteristics with MCM2 and TIP30 expression in SC/ASC and AC. As presented in Table I, the percentage of cases in patients older 
Table I. Comparison of gallbladder SC/ASC and AC clinicopathological features and MCM2 and TIP30 expression status.

\begin{tabular}{|c|c|c|c|c|}
\hline Clinicopathological characteristic & $\mathrm{SC}(\mathrm{n}=46)$ & $\operatorname{ASC}(n=80)$ & $\chi^{2}$ & P-value \\
\hline \multicolumn{5}{|l|}{ Sex } \\
\hline Male & $19(41.3)$ & $26(32.5)$ & \multirow[t]{2}{*}{0.986} & \multirow[t]{2}{*}{0.352} \\
\hline Female & $27(58.7)$ & $54(67.5)$ & & \\
\hline \multicolumn{5}{|l|}{ Age (year) } \\
\hline$\leq 45$ & $3(6.5)$ & $16(20.0)$ & \multirow[t]{2}{*}{4.143} & \multirow[t]{2}{*}{0.042} \\
\hline$>45$ & $43(93.5)$ & $64(80.0)$ & & \\
\hline \multicolumn{5}{|l|}{ Differentiation } \\
\hline Well-differentiated & $16(34.8)$ & $27(33.8)$ & \multirow{3}{*}{8.515} & \multirow{3}{*}{0.014} \\
\hline Moderately differentiated & $24(52.2)$ & $25(31.3)$ & & \\
\hline Poorly differentiated & $6(13.0)$ & $28(35.0)$ & & \\
\hline \multicolumn{5}{|l|}{ Maximum diameter of tumor $(\mathrm{cm})$} \\
\hline$\leq 3 \mathrm{~cm}$ & $20(43.5)$ & $50(62.5)$ & \multirow[t]{2}{*}{4.280} & \multirow[t]{2}{*}{0.039} \\
\hline$>3 \mathrm{~cm}$ & $26(56.5)$ & $30(37.5)$ & & \\
\hline \multicolumn{5}{|l|}{ Cholecystolithiasis } \\
\hline$(-)$ & $18(39.1)$ & $42(52.5)$ & \multirow[t]{2}{*}{2.093} & \multirow[t]{2}{*}{0.148} \\
\hline$(+)$ & $28(60.9)$ & $38(47.5)$ & & \\
\hline \multicolumn{5}{|l|}{ TNM stages } \\
\hline $\mathrm{I}+\mathrm{II}$ & $12(26.1)$ & $21(26.3)$ & \multirow{3}{*}{0.287} & \multirow{3}{*}{0.866} \\
\hline III & $20(33.5)$ & $38(47.5)$ & & \\
\hline IV & $14(30.4)$ & $21(26.3)$ & & \\
\hline \multicolumn{5}{|l|}{ Lymph node metastasis } \\
\hline$(-)$ & $17(37.0)$ & $30(37.5)$ & \multirow[t]{2}{*}{0.004} & \multirow[t]{2}{*}{0.952} \\
\hline$(+)$ & $29(63.0)$ & $50(62.5)$ & & \\
\hline \multicolumn{5}{|l|}{ Locoregional invasion } \\
\hline$(-)$ & $16(34.8)$ & $31(38.8)$ & \multirow[t]{2}{*}{0.197} & \multirow[t]{2}{*}{0.658} \\
\hline$(+)$ & $30(62.5)$ & $49(61.3)$ & & \\
\hline \multicolumn{5}{|l|}{ Operation methods } \\
\hline Radical & $14(30.4)$ & $26(32.5)$ & \multirow{3}{*}{0.215} & \multirow{3}{*}{0.898} \\
\hline Palliative & $18(39.1)$ & $28(35.0)$ & & \\
\hline Without resection & $14(30.4)$ & $26(32.5)$ & & \\
\hline Mean survival time & $10.07(4-25)$ & $10.34(3-27)$ & 0.014 & 0.906 \\
\hline \multicolumn{5}{|l|}{ MCM2 } \\
\hline$(-)$ & $24(52.2)$ & $38(47.5)$ & 0.951 & 0.382 \\
\hline$(+)$ & $22(47.8)$ & $42(52.5)$ & & \\
\hline TIP30 & & & & \\
\hline$(-)$ & $26(56.5)$ & $38(47.5)$ & 0.289 & 0.678 \\
\hline$(+)$ & $20(43.5)$ & $42(52.5)$ & & \\
\hline
\end{tabular}

SC, squamous cell; ASC, adenosquamous carcinoma; MCM2, minichromosome maintenance complex component 2; TIP30, HIV-1 tat interactive protein 2 .

than 45 years and with a tumor mass $>3 \mathrm{~cm}$ was significantly higher in the SC/ASC group compared with the ACs $(\mathrm{P}<0.05)$. In contrast, the percentage of poorly differentiated tumors was significantly reduced in SC/ASC compared with AC. No significant differences in other clinicopathological characteristics (including gender, TNM stage, invasion, lymph node metastasis, history of gallstones, operative procedure and average survival time) were observed between the SC/ASC and AC groups. Immunohistochemistry identified that MCM2-positive cells exhibited brown nuclear staining, whereas TIP30-positive cells presented with a brown membrane and cytoplasmic staining in the SC/ASC (Fig. 1) and AC (Fig. 2) tissues. In addition, no significant differences in the percentage of positive MCM2 and TIP30 expression was observed between patients with SC/ASC and AC (Table I).

The association of MCM2 and TIP30 expression with GBC clinicopathological characteristics. As presented in Table II, 


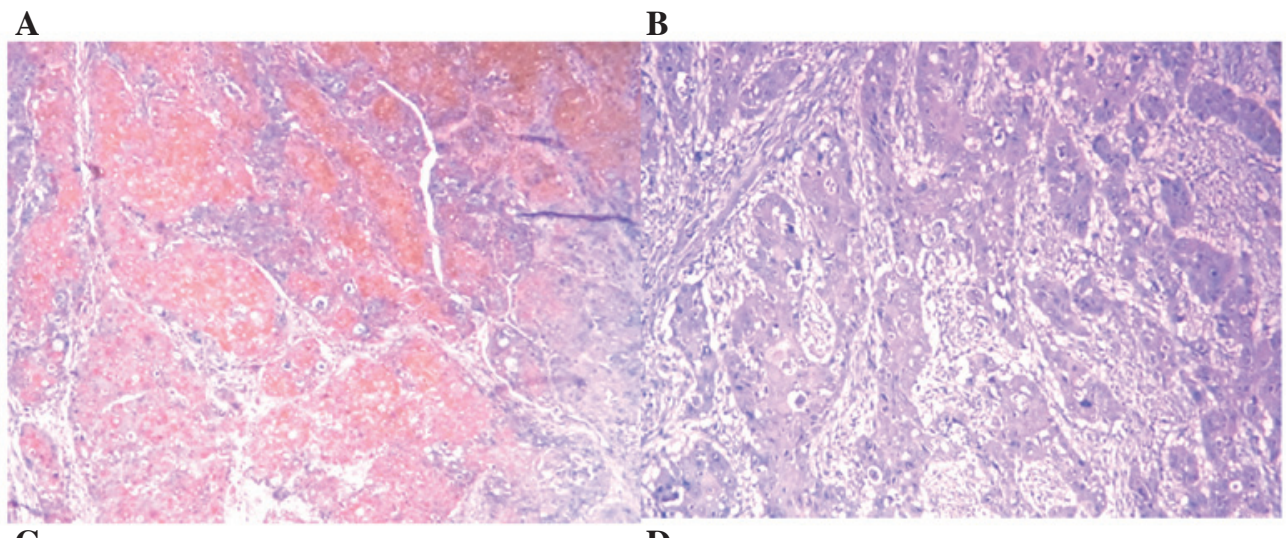

C

D

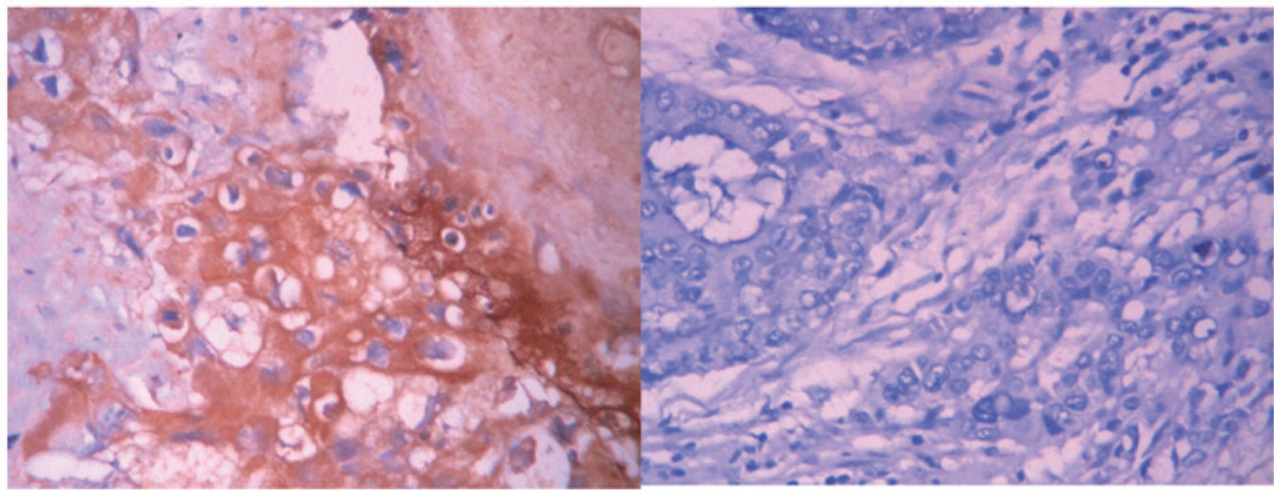

Figure 1. MCM2 and TIP30 expression in SC/ASC. EnVision immunohistochemistry, original magnification x200. MCM2 and TIP30 positive staining was mainly localized in the cytoplasm. (A) Positive MCM2 expression in poorly differentiated SC/ASC. (B) Negative MCM2 expression in moderately differentiated SC/ASC. (C) Positive TIP30 expression in well differentiated SC/ASC. (D) Negative TIP30 expression in moderately differentiated SC/ASC. MCM2, minichromosome maintenance complex component 2; TIP30, HIV-1 tat interactive protein 2; SC, squamous cell; ASC, adenosquamous carcinoma.

A

B

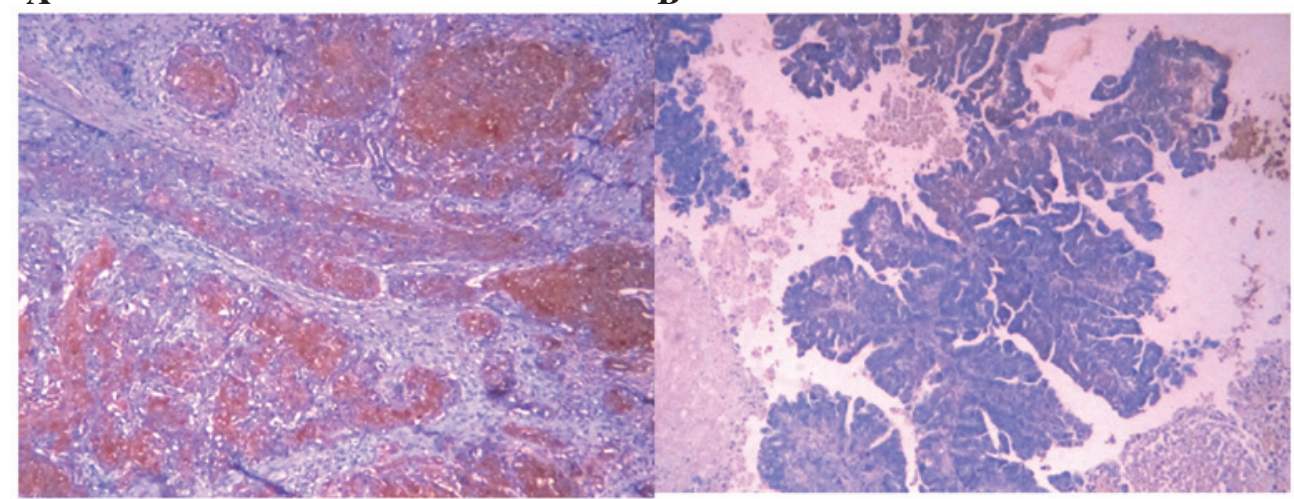

C

D

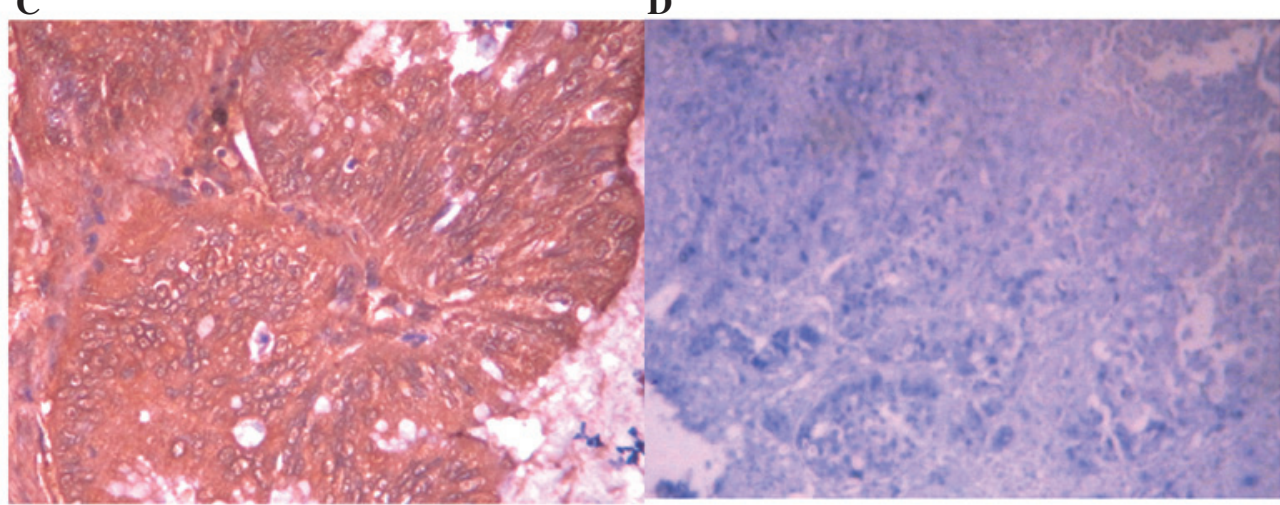

Figure 2. MCM2 and TIP30 expression in AC. EnVision immunohistochemistry, original magnification x200. MCM2 and TIP30 positive staining was mainly localized in the cytoplasm. (A) Positive MCM2 expression in poorly differentiated AC. (B) Negative MCM2 expression in well differentiated AC. (C) Positive TIP30 expression in well differentiated AC. (D) Negative TIP30 expression in poorly differentiated AC. MCM2, minichromosome maintenance complex component 2; TIP30, HIV-1 tat interactive protein 2; AC, adenocarcinoma. 
Table II. The association of MCM2 and TIP30 expression with the clinicopathological characteristics of SC/ASC.

\begin{tabular}{|c|c|c|c|c|c|c|c|}
\hline \multirow{2}{*}{$\begin{array}{l}\text { Clinicalpathological } \\
\text { characteristics }\end{array}$} & \multirow[b]{2}{*}{ Total no. } & \multicolumn{3}{|c|}{ MCM2 } & \multicolumn{3}{|c|}{ TIP30 } \\
\hline & & Pos no. $(\%)$ & $\chi^{2}$ & P-value & Pos no. $(\%)$ & $\chi^{2}$ & P-value \\
\hline Sex & & & 0.090 & 0.765 & & 1.565 & 0.211 \\
\hline Male & 19 & $9(47.4)$ & & & $12(63.2)$ & & \\
\hline Female & 27 & $14(51.9)$ & & & $12(44.6)$ & & \\
\hline Age (years) & & & 0.357 & 0.550 & & 0.270 & 0.603 \\
\hline$\leq 45$ & 3 & $1(33.3)$ & & & $2(66.7)$ & & \\
\hline$>45$ & 43 & $22(51.2)$ & & & $22(51.2)$ & & \\
\hline Pathological type & & & 0.354 & 0.552 & & 2.333 & 0.127 \\
\hline $\mathrm{SC}$ & 26 & $14(53.8)$ & & & $11(42.3)$ & & \\
\hline ASC & 20 & $9(45.0)$ & & & $13(65.0)$ & & \\
\hline Differentiation & & & 7.167 & 0.028 & & 3.753 & 0.153 \\
\hline Well & 16 & $6(37.5)$ & & & $10(62.5)$ & & \\
\hline Moderately & 24 & $11(45.8)$ & & & $13(54.2)$ & & \\
\hline Poorly & 6 & $6(100.0)$ & & & $1(16.7)$ & & \\
\hline Tumor mass size & & & 5.662 & 0.017 & & 7.389 & 0.007 \\
\hline$\leq 3 \mathrm{~cm}$ & 20 & $6(30.0)$ & & & $15(75.0)$ & & \\
\hline$>3 \mathrm{~cm}$ & 26 & $167(65.4)$ & & & $9(34.6)$ & & \\
\hline Gallstones & & & 0.365 & 0.546 & & 0.708 & 0.400 \\
\hline No & 18 & $10(55.6)$ & & & $8(44.4)$ & & \\
\hline Yes & 28 & $13(46.4)$ & & & $16(57.1)$ & & \\
\hline TNM stage & & & 10.943 & 0.004 & & 9.936 & 0.008 \\
\hline $\mathrm{I}+\mathrm{II}$ & 12 & $3(25.0)$ & & & $10(83.3)$ & & \\
\hline III & 20 & $8(40.0)$ & & & $11(55.0)$ & & \\
\hline IV & 14 & $12(85.7)$ & & & $3(21.4)$ & & \\
\hline Lymph metastasis & & & 4.572 & 0.032 & & 6.379 & 0.012 \\
\hline No & 17 & $5(29.4)$ & & & $13(76.5)$ & & \\
\hline Yes & 29 & $18(62.1)$ & & & $11(37.9)$ & & \\
\hline Invasion & & & 6.133 & 0.013 & & 12.270 & 0.000 \\
\hline No & 16 & $4(25.0)$ & & & $14(87.5)$ & & \\
\hline Yes & 30 & $19(63.3)$ & & & $10(33.3)$ & & \\
\hline Surgery & & & 7.365 & 0.025 & & 9.296 & 0.00 \\
\hline Radical & 14 & $3(21.4)$ & & & $11(78.6)$ & & \\
\hline Palliative & 18 & $10(55.6)$ & & & $10(55.6)$ & & \\
\hline Biopsy & 14 & $10(71.4)$ & & & $3(21.4)$ & & \\
\hline
\end{tabular}

MCM2, minichromosome maintenance complex component 2; TIP30, HIV-1 tat interactive protein 2; SC, squamous cell; ASC, adenosquamous carcinoma.

the percentage of positive MCM2 and negative TIP30 expression in tumor tissues was significantly associated with large tumor size, high TNM stage, invasion, lymph node metastasis and no surgical curability of SC/ASC $(\mathrm{P}<0.05$ or $\mathrm{P}<0.01)$. Positive MCM2 expression was significantly associated with poor differentiation in SC/ASC $(\mathrm{P}<0.05)$. There was no significant association between MCM2 and TIP30 and pathological type, gender, age and history of gallstones.

As presented in Table III, the percentage of positive MCM2 and negative TIP30 expression in AC tumors was significantly higher in cases with poor differentiation, larger tumor mass, higher TMN stage with lymph node metastasis, invasion to the tissues and organs surrounding the gallbladder and lack of surgical curability when compared with cases that were well differentiated, had a small tumor mass, lower TMN stage, no lymph metastasis, no invasion and that were radically resected $(\mathrm{P}<0.05, \mathrm{P}<0.01)$. There was no association between MCM2 and TIP30 and gender, age and history of gallstones.

The correlation of MCM2 and/or TIP30 expression with survival in patients with SC/ASC and AC. Survival information 
Table III. The association of MCM2 and TIP30 expression with the clinicopathological characteristics of AC.

\begin{tabular}{|c|c|c|c|c|c|c|c|}
\hline \multirow{2}{*}{$\begin{array}{l}\text { Clinicopathological } \\
\text { characteristics }\end{array}$} & \multirow[b]{2}{*}{ Total no. } & \multicolumn{3}{|c|}{ MCM2 } & \multicolumn{3}{|c|}{ TIP30 } \\
\hline & & Pos no. $(\%)$ & $\chi^{2}$ & P-value & Pos no. (\%) & $\chi^{2}$ & P-value \\
\hline Sex & & & 0.000 & 0.990 & & 0.024 & 0.877 \\
\hline Male & 26 & $14(53.8)$ & & & $13(50.0)$ & & \\
\hline Female & 54 & $29(53.7)$ & & & $26(48.1)$ & & \\
\hline Age (years) & & & 2.124 & 0.145 & & 3.202 & 0.074 \\
\hline$\leq 45$ & 16 & $6(37.5)$ & & & $11(68.8)$ & & \\
\hline$>45$ & 64 & $37(57.8)$ & & & $28(43.8)$ & & \\
\hline Differentiation & & & 7.880 & 0.019 & & 10.784 & 0.004 \\
\hline Well & 27 & $11(40.7)$ & & & $20(74.1)$ & & \\
\hline Moderately & 25 & $11(44.0)$ & & & $10(40.0)$ & & \\
\hline Poorly & 28 & $21(75.0)$ & & & $9(32.1)$ & & \\
\hline Tumor mass size & & & 5.099 & 0.024 & & 6.754 & 0.009 \\
\hline$\leq 3 \mathrm{~cm}$ & 50 & $22(44.0)$ & & & $30(60.0)$ & & \\
\hline$>3 \mathrm{~cm}$ & 30 & $21(70.0)$ & & & $9(30.0)$ & & \\
\hline Gallstones & & & 1.337 & 0.248 & & 1.279 & 0.258 \\
\hline No & 42 & 20 (47.6) & & & $23(54.8)$ & & \\
\hline Yes & 38 & $23(60.5)$ & & & $16(42.1)$ & & \\
\hline TNM stage & & & 11.943 & 0.003 & & 7.328 & 0.026 \\
\hline $\mathrm{I}+\mathrm{II}$ & 21 & $8(38.1)$ & & & 13 (61.9) & & \\
\hline III & 38 & $17(44.7)$ & & & $21(55.3)$ & & \\
\hline IV & 21 & $18(85.7)$ & & & $5(23.8)$ & & \\
\hline Lymph metastasis & & & 5.635 & 0.018 & & 4.086 & 0.043 \\
\hline No & 30 & $11(36.7)$ & & & $19(63.3)$ & & \\
\hline Yes & 50 & $32(64.0)$ & & & $20(40.0)$ & & \\
\hline Invasion & & & 4.605 & 0.036 & & 5.035 & 0.025 \\
\hline No & 31 & $12(38.7)$ & & & $20(64.5)$ & & \\
\hline Yes & 49 & $31(63.3)$ & & & $19(38.8)$ & & \\
\hline Surgery & & & 8.665 & 0.013 & & 7.449 & 0.024 \\
\hline Radical & 26 & $10(38.5)$ & & & $16(61.5)$ & & \\
\hline Palliative & 28 & $13(46.4)$ & & & $16(57.1)$ & & \\
\hline Biopsy & 26 & $20(76.9)$ & & & $7(26.9)$ & & \\
\hline
\end{tabular}

MCM2, minichromosome maintenance complex component 2; TIP30, HIV-1 tat interactive protein 2; AC, adenocarcinoma.

for the 46 patients with SC/ASC was obtained through letters and phone calls. The follow-up time was 2 years, and patients who survived longer than 2 years were included in the analysis as censored cases. A total of 13 patients survived $>1$ year (of which 4 survived $>2$ years), and 33 patients survived $<1$ year, with a mean survival time of $10.07 \pm 0.78$ months. Kaplan-Meier survival analysis demonstrated that differentiation, tumor size, TNM stage, lymph node metastasis, invasion and the operative procedure were significantly associated $(\mathrm{P}<0.001)$ with mean survival time in SC/ASC patients (Table IV). The mean survival time of MCM2-positive patients was significantly lower compared with MCM2-negative patients $(\mathrm{P}<0.001)$. In contrast, the mean survival time of TIP30-positive patients was significantly greater than TIP30-negative patients $(\mathrm{P}<0.001)$ (Table IV; Fig. 3). Cox multivariate analysis indicated that tumor size $\geq 3 \mathrm{~cm}$, TMN stage, lymph node metastasis, invasion, operative procedure and MCM2-positive expression or TIP30-negative expression were negatively correlated with post-operative survival, and positively correlated with mortality. This suggests that MCM2 positivity is a risk factor, and that TIP30 is a prognostic indicator in SC/ASC (Table V).

The survival information of 80 patients with $\mathrm{AC}$ was also obtained. A total of 23 patients survived $>1$ year (of which 9 survived $>2$ years), and 57 survived $<1$ year; the mean survival time was $10.34 \pm 0.63$ months. Kaplan-Meier survival analysis indicated that differentiation, tumor size, TNM stage, lymph node metastasis, invasion and operative procedure were significantly associated $(\mathrm{P}<0.001)$ with the mean survival time of patients with AC (Table VI). The mean survival time of MCM2-positive patients with $\mathrm{AC}$ was significantly lower 
Table IV. Association between MCM2 and TIP30 expression, clinicopathological characteristics and average survival of SC/ASC patients.

\begin{tabular}{|c|c|c|c|c|}
\hline $\begin{array}{l}\text { Clinicopathological } \\
\text { characteristics }\end{array}$ & Samples (n) & $\begin{array}{l}\text { Average survival } \\
\text { (month) }\end{array}$ & Chi-square & P-value \\
\hline Sex & & & 0.767 & 0.381 \\
\hline Male & 19 & 10.74 (6-24) & & \\
\hline Female & 27 & $9.85(4-24)$ & & \\
\hline Age (years) & & & 2.023 & 0.155 \\
\hline$\leq 45$ & 3 & $15.67(8-24)$ & & \\
\hline$>45$ & 56 & $9.84(4-25)$ & & \\
\hline Pathological type & & & 0.223 & 0.637 \\
\hline $\mathrm{SC}$ & 26 & $10.19(4-24)$ & & \\
\hline ASC & 20 & $10.25(4-24)$ & & \\
\hline Differentiation & & & 19.125 & 0.000 \\
\hline Well & 16 & $13.81(5-24)$ & & \\
\hline Moderately & 24 & $8.92(4-18)$ & & \\
\hline Poorly & 6 & $5.83(4-9)$ & & \\
\hline Tumor mass size & & & 31.337 & 0.000 \\
\hline$\leq 3 \mathrm{~cm}$ & 20 & $14.35(7-24)$ & & \\
\hline$>3 \mathrm{~cm}$ & 26 & $7.04(4-11)$ & & \\
\hline Gallstones & & & 3.730 & 0.053 \\
\hline No & 18 & $8.22(4-12)$ & & \\
\hline Yes & 28 & $11.50(4-24)$ & & \\
\hline TNM stage & & & 51.139 & 0.000 \\
\hline $\mathrm{I}+\mathrm{II}$ & 12 & $17.00(9-24)$ & & \\
\hline III & 20 & $9.20(7-15)$ & & \\
\hline IV & 14 & $5.86(4-8)$ & & \\
\hline Lymph node metastasis & & & 16.219 & 0.000 \\
\hline No & 17 & $14.24(4-24)$ & & \\
\hline Yes & 29 & $7.86(4-15)$ & & \\
\hline Invasion & & & 32.271 & 0.000 \\
\hline No & 16 & $15.75(9-24)$ & & \\
\hline Yes & 30 & $7.27(4-12)$ & & \\
\hline Surgery & & & 50.165 & 0.000 \\
\hline Radical & 14 & $16.64(10-24)$ & & \\
\hline Palliative & 18 & $8.50(6-12)$ & & \\
\hline Biopsy & 14 & $6.00(4-8)$ & & \\
\hline MCM2 & & & 17.096 & 0.000 \\
\hline- & 23 & $13.17(6-24)$ & & \\
\hline+ & 23 & $7.26(4-12)$ & & \\
\hline TIP30 & & & 21.434 & 0.000 \\
\hline- & 22 & $7.05(4-12)$ & & \\
\hline+ & 24 & $13.13(6-24)$ & & \\
\hline
\end{tabular}

MCM2, minichromosome maintenance complex component 2; TIP30, HIV-1 tat interactive protein 2; SC, squamous cell; ASC, adenosquamous carcinoma.

than that of MCM2-negative patients with $\mathrm{AC}(\mathrm{P}=0.001)$, whereas the mean survival time of TIP30-positive patients was significantly higher than TIP30-negative patients $(\mathrm{P}=0.009)$ (Table VI; Fig. 4). Cox multivariate analysis demonstrated that differentiation, tumor size $\geq 3 \mathrm{~cm}$, TMN stage, lymph node metastasis, invasion, operative procedure and MCM2-positive or TIP30-negative expression were positively correlated with poor survival in patients with AC (Table VII). 
Table V. Multivariate Cox regression analysis of survival rate in SC/ASC patients.

$95 \%$ confidence interval

\begin{tabular}{lcccccccc}
\cline { 5 - 8 } Groups & Factors & RC & SE & Wald & P & RR & Lower & Upper \\
\hline Pathological types & SC/ASC & 0.108 & 0.360 & 0.090 & 0.764 & 1.114 & 0.550 & 2.256 \\
Differatiation & Well/moderately/poorly & 1.100 & 0.399 & 7.600 & 0.006 & 3.004 & 1.374 & 6.567 \\
Tumor mass size & $\leq 3 />3 \mathrm{~cm}$ & 2.351 & 0.791 & 8.834 & 0.003 & 10.496 & 2.227 & 49.470 \\
Gallstone & No/yes & 0.859 & 0.479 & 3.216 & 0.073 & 2.361 & 0.923 & 6.037 \\
TNM stage & I+II/III/IV & 1.252 & 0.518 & 5.842 & 0.016 & 3.497 & 1.267 & 9.653 \\
Lymph metastasis & No/yes & 1.180 & 0.473 & 6.224 & 0.013 & 3.254 & 1.288 & 8.224 \\
Invasion & No/yes & 2.624 & 0.809 & 10.520 & 0.001 & 13.791 & 2.825 & 67.332 \\
Surgery & Radical/palliative/ & 1.084 & 0.467 & 5.388 & 0.020 & 2.956 & 1.184 & 7.384 \\
MCM2 & biopsy & & & & & & & \\
TIP30 & $-/+$ & 1.136 & 0.488 & 5.419 & 0.020 & 3.114 & 1.197 & 8.105 \\
\hline & $-/+$ & -0.981 & 0.470 & 4.357 & 0.037 & 0.375 & 0.149 & 0.942 \\
\hline
\end{tabular}

SC, squamous cell; ASC, adenosquamous carcinoma; RC, regression coefficients; SE, standard error; RR, relative risk; MCM2, minichromosome maintenance complex component 2; TIP30, HIV-1 tat interactive protein 2.

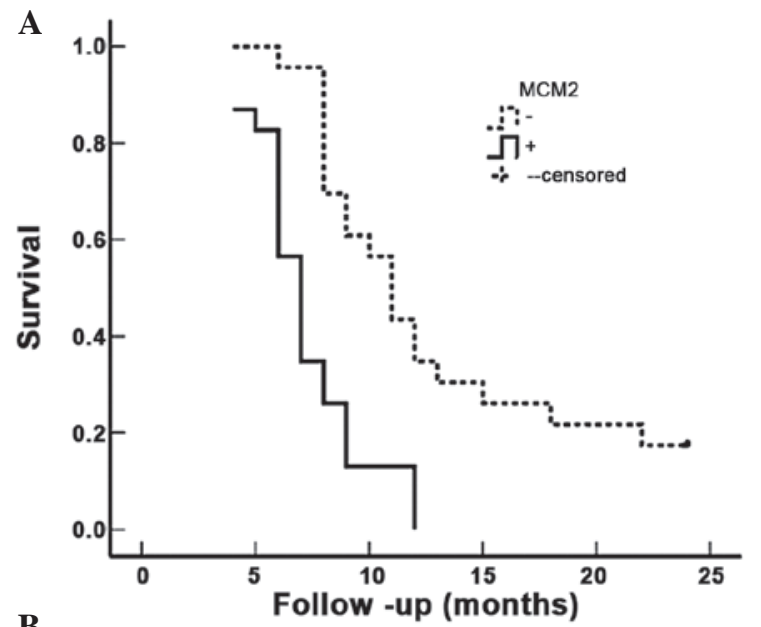

B

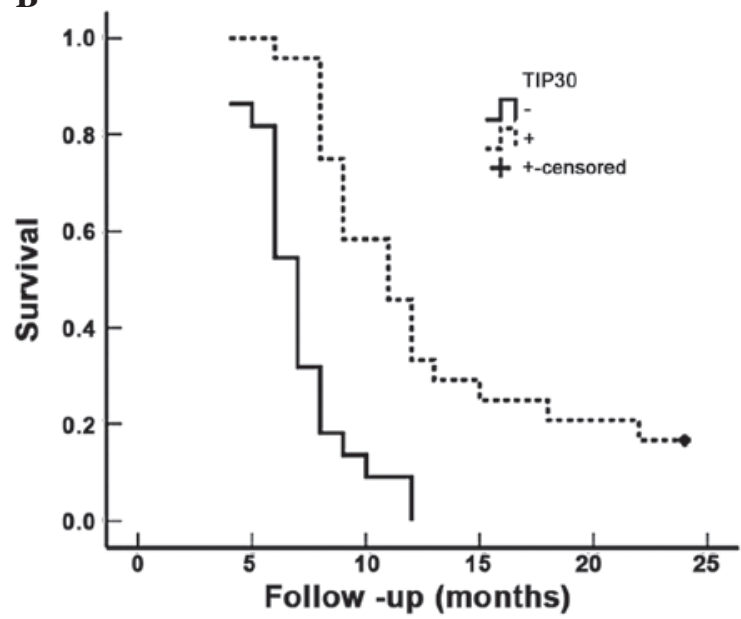

Figure 3. MCM2 and TIP30 expression and survival in patients with SC/ASC of the gallbladder. (A) Kaplan-Meier plots of overall survival in patients with SC/ASC and with MCM2 positive and negative expression. (B) Kaplan-Meier plots of overall survival in patients with SC/ASC and with TIP30 positive and negative expression. MCM2, minichromosome maintenance complex component 2; TIP30, HIV-1 tat interactive protein 2; SC, squamous cell; ASC, adenosquamous carcinoma.

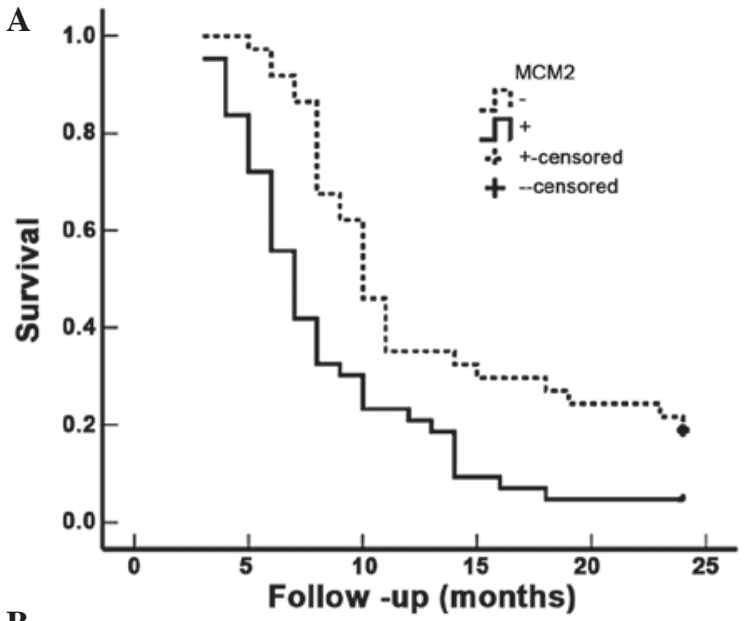

B

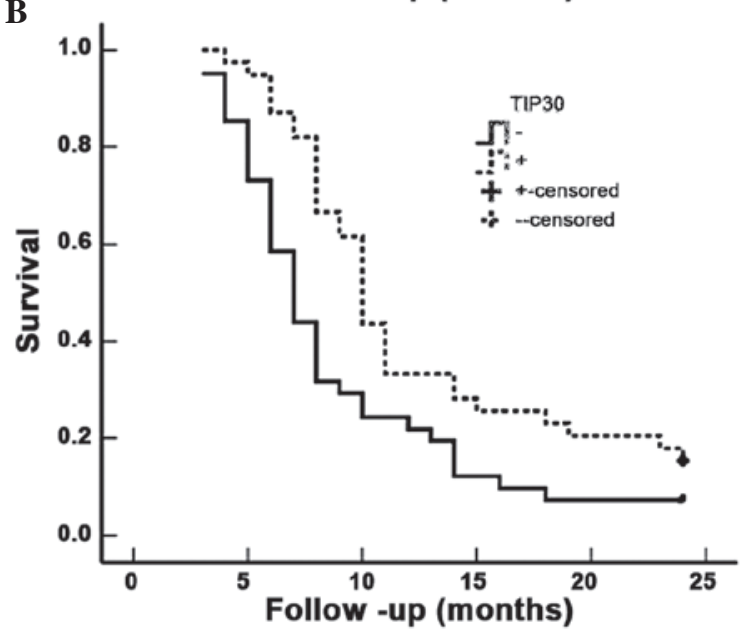

Figure 4. MCM2 and TIP30 expression levels and survival in patients with AC of the gallbladder. (A) Kaplan-Meier plots of overall survival in patients with AC and with MCM2 positive and negative expression. (B) Kaplan-Meier plots of overall survival in patients with AC and with TIP30 positive and negative expression. MCM2, minichromosome maintenance complex component 2; TIP30, HIV-1 tat interactive protein 2; AC, adenocarcinoma. 
Table VI. Association between MCM2 and TIP30 expression, clinicopathological characteristics and average survival of AC patients.

\begin{tabular}{|c|c|c|c|c|}
\hline $\begin{array}{l}\text { Clinicopathological } \\
\text { characteristics }\end{array}$ & Samples (n) & $\begin{array}{l}\text { Average survival } \\
\text { (month) }\end{array}$ & Chi-square & P-value \\
\hline Sex & & & 2.567 & 0.109 \\
\hline Male & 26 & $9.58(3-24)$ & & \\
\hline Female & 54 & $11.30(3-24)$ & & \\
\hline Age (years) & & & 0.003 & 0.956 \\
\hline$\leq 45$ & 16 & $10.81(4-24)$ & & \\
\hline$>45$ & 64 & $10.72(3-24)$ & & \\
\hline Differentiation & & & 32.501 & 0.000 \\
\hline Well & 27 & $15.07(5-24)$ & & \\
\hline Moderately & 25 & $10.60(4-24)$ & & \\
\hline Poorly & 28 & $6.68(3-14)$ & & \\
\hline Tumor mass size & & & 68.283 & 0.000 \\
\hline$\leq 3 \mathrm{~cm}$ & 50 & $13.70(6-24)$ & & \\
\hline$>3 \mathrm{~cm}$ & 30 & $5.80(3-10)$ & & \\
\hline Gallstones & & & 0.246 & 0.620 \\
\hline No & 42 & $10.19(3-24)$ & & \\
\hline Yes & 38 & $11.34(4-24)$ & & \\
\hline TNM stage & & & 105.825 & 0.000 \\
\hline $\mathrm{I}+\mathrm{II}$ & 21 & $18.96(5-24)$ & & \\
\hline III & 38 & $9.29(6-15)$ & & \\
\hline IV & 21 & $5.14(3-7)$ & & \\
\hline Lymph node metastasis & & & 42.372 & 0.000 \\
\hline No & 30 & $16.27(4-24)$ & & \\
\hline Yes & 50 & $7.42(3-14)$ & & \\
\hline Invasion & & & 55.535 & 0.000 \\
\hline No & 31 & $16.68(7-24)$ & & \\
\hline Yes & 49 & $6.98(3-11)$ & & \\
\hline Surgery & & & 113.141 & 0.000 \\
\hline Radical & 26 & $18.31(10-24)$ & & \\
\hline Palliative & 28 & $8.64(6-11)$ & & \\
\hline Biopsy & 26 & $5.42(3-9)$ & & \\
\hline MCM2 & & & 11.466 & 0.001 \\
\hline- & 37 & $13.24(5-24)$ & & \\
\hline+ & 43 & $8.58(3-24)$ & & \\
\hline TIP30 & & & 6.894 & 0.009 \\
\hline- & 41 & $8.95(3-24)$ & & \\
\hline+ & 39 & $12.62(4-24)$ & & \\
\hline
\end{tabular}

MCM2, minichromosome maintenance complex component 2; TIP30, HIV-1 tat interactive protein 2; AC, adenocarcinoma.

\section{Discussion}

Previous studies suggested that squamous carcinoma proliferates at a higher rate than AC, however lymph node metastasis is less common with squamous tumors $(26,27)$. The results of the current study observed no differences in invasion and lymph node metastasis between AC and SC/ASC, however an increased number of patients with SC/ASC had an increased tumor size. There were also no significant differences in differentiation, TNM stage, surgical curability and post-operative survival time between patients with $\mathrm{AC}$ (10.34 \pm 0.63 months) and SC/ASC (10.07 \pm 0.78 months). These observations suggest that squamous differentiation is no more aggressive than glandular differentiation in the gallbladder. In addition, it was observed that radical resection was a good prognostic factor in patients with $\mathrm{AC}$ and SC/ASC. However, $86 \%$ of patients with SC/ASC and $74 \%$ with $\mathrm{AC}$ were diagnosed at an inoperable stage. Inconsistent 
Table VII. Multivariate Cox regression analysis of survival rate in patients with AC.

$95 \%$ confidence interval

\begin{tabular}{lccccccrr}
\cline { 5 - 8 } Groups & Factors & $\mathrm{RC}$ & $\mathrm{SE}$ & Wald & $\mathrm{P}$ & $\mathrm{RR}$ & Lower & Upper \\
\hline Differatiation & Well/moderately/poorly & 1.364 & 0.514 & 7.042 & 0.008 & 3.912 & 1.428 & 10.713 \\
Tumor mass size & $\leq 3 />3 \mathrm{~cm}$ & 1.290 & 0.430 & 9.000 & 0.003 & 3.633 & 1.564 & 8.438 \\
Gallstone & No/yes & 0.151 & 0.270 & 0.313 & 0.576 & 1.163 & 0.685 & 1.974 \\
TNM stage & I+II/III/IV & 1.194 & 0.469 & 6.481 & 0.011 & 3.300 & 1.316 & 8.275 \\
Lymph metastasis & No/yes & 1.059 & 0.446 & 5.638 & 0.018 & 2.883 & 1.203 & 6.911 \\
Invasion & No/yes & 1.386 & 0.506 & 7.503 & 0.006 & 3.999 & 1.483 & 10.781 \\
Surgery & Radical/palliative/biopsy & 1.426 & 0.479 & 8.863 & 0.003 & 4.162 & 1.628 & 10.642 \\
MCM2 & $-/+$ & 0.995 & 0.393 & 6.410 & 0.011 & 2.705 & 1.252 & 5.843 \\
TIP30 & $-/+$ & -0.857 & 0.374 & 5.251 & 0.022 & 0.424 & 0.204 & 0.883 \\
\hline
\end{tabular}

AC, adenocarcinoma; RC, regression coefficients; SE, standard error; RR, relative risk; MCM2, minichromosome maintenance complex component 2; TIP30, HIV-1 tat interactive protein 2.

with a previous report concluding that SC/ASC occurs predominantly in females $(\mathrm{F} / \mathrm{M}=3.8)(6)$, no difference in the prevalence of SC/ASC between females and males (F/M ratio=1.4) was observed in the current study. However, the prevalence of SC/ASC among older patients was increased compared with AC. Consistent with previous reports $(2,6)$, it was observed that $4.34 \%$ of GBCs were SC/ASC. This suggests that the clinicopathological presentation of SC/ASC was not significantly different from AC. Current knowledge of the clinicopathological characteristics of SC/ASC was obtained predominantly from individual case reports or the analysis of small case series. Therefore, the present study provided more accurate information describing the differences between the rare SC/ASC subtype and the more common AC.

Accumulating evidence has demonstrated that low levels of expression of TIP30 and increased expression of MCM2 correspond with increased malignancy of tumors, high incidence of metastasis, poor prognosis and high invasive potential (28-31). In the present study, an extensive collection of human gallbladder SC/ASC and AC samples was used to demonstrate that elevated MCM2 or lowered TIP30 levels are important prognostic factors, independent of other clinicopathological factors.

MCM2 is a key protein that regulates the transition of a cell from $G_{1}$ phase to $S$ phase; its expression is silenced in mature cells or those in $\mathrm{G}_{0}$. Therefore, MCM2 is a specific marker that indicates an active state of cell proliferation $(28,29)$. TIP30 is a serine/threonine kinase that blocks cell function at $G_{0} / G_{1}$ phase, which subsequently reduces the proportion of cells in $\mathrm{S}$ phase. However, the clinicopathological characteristics of these proteins in GBC remain to be fully elucidated. Previous studies reported that MCM levels could reflect tumor carcinogenesis and progression $(9,14)$. Because of its high specificity and sensitivity for determining cell proliferation, MCM2 may be a malignant marker. MCM2 has been used for population screening for neoplasms for a range of benign lesions using exfoliated cells (32-34). Positive MCM2 and negative TIP30 expression were significantly associated with large tumor size, high TNM stage, invasion, lymph node metastasis, and no surgical curability (only biopsy) of SC/ASC and AC. Therefore, high expression of MCM2 and low expression of TIP30 may be used as a biological marker to identify subgroups of patients who may require more aggressive treatment. Patients with high MCM2 and low TIP30 expression in their tumors are more likely to suffer from metastatic recurrence. These patients may need to be monitored closely for clinical signs of relapse to ensure that therapeutic inventions are applied early enough for optimal outcomes. It is reasonable to predict that the combination of MCM2(+) and TIP30(-) may be a powerful predictor for malignant metastasis and a biomarker for the early diagnosis and grading of GBC.

Previous studies demonstrated that MCM levels may reflect clinical behavior and prognosis of cancer $(9,14)$. In the current study, Kaplan-Meier survival analysis indicated that the survival time of patients with positive expression of MCM2 was significantly shorter than that of patients with negative expression; Cox multivariate analysis indicated that MCM2 expression was positively correlated with mortality. Conversely, patients expressing TIP30 survived longer than those with negative expression of TIP30; Cox multivariate analysis identified that TIP30 expression was positively correlated with post-operative survival. Therefore, MCM2 and TIP30 are proposed as two independent prognostic factors.

The surgical removal of the gallbladder with dissection of part of the liver and the lymph nodes is currently the most common treatment for resectable GBC. However, the majority of patients present with poor prognosis and will experience a survival time of $<1$ year. Due to the fact that palliative chemotherapy and radiation therapy offer little benefit in GBC (2), there is an urgent requirement for novel therapeutic approaches to improve patient survival. Malignant tumors with high expression of MCM2 have an increased rate of progression, are prone to metastasis and invasion and have 
poor prognosis. TIP30 inhibits tumor growth and metastasis by regulating the expression of cell proliferation-, apoptosis-, and angiogenesis-associated genes $(35,36)$. High expression of MCM2 and low expression of TIP30 in gallbladder tumor cells may allow them to be targeted for gene therapy. There are several possible pathways for the development of novel treatments for GBC based on the molecular targeting of MCM2 and TIP30. For example, viral- or non viral-based delivery (such as the nanoparticle-based delivery of small interfering RNA) could be used to silence MCM2 expression alone or in combination with the overexpression of TIP30. Notably, MCM proteins are specifically required in cycling cells, however are absent from quiescent cells. Therefore, using the MCM2 promoter to drive the expression of TIP30 or other apoptotic genes may provide tumor-specific targeting in gallbladder $\mathrm{AC}$ or other tumor types that express $\mathrm{MCM}$ proteins.

In conclusion, reduced expression of TIP30 and elevated expression of MCM2 are important markers for the progression, clinical biological behavior, and prognosis of gallbladder SC/ASC and AC. Measuring MCM2 and TIP30 expression may be a tool for the early detection of GBC in benign lesions in addition to in population screening. The observation that MCM proteins are required only in cycling cells and are not expressed in quiescent cells, in addition to the role of TIP30 in inhibiting tumor growth, suggests that these two markers have potential to be developed as targets for gene therapy by silencing MCM2 expression and/or overexpressing TIP30.

\section{Acknowledgements}

The current study was supported by the Department of Pathology, Basic School of Medicine (Changsha, China); Department of Pathology, Second Xiangya Hospital (Changsha, China); Department of Pathology, Third Xiangya Hospital (Changsha, China); Department of Pathology, Loudi Central Hospital (Loudi, China); and the Department of Pathology, Hunan Provincial Tumor Hospital (Changsha, China). The present study was supported by a grant from the National Natural Science Foundation of China (grant no. 81472738).

\section{References}

1. Jemal A, Siegel R, Ward E, Hao Y, Xu J, Murray T and Thun MJ: Cancer statistics, 2008. CA Cancer J Clin 58: 71-96, 2008.

2. Jayaraman S and Jarnagin WR: Management of gallbladder cancer. Gastroenterol Clin North Am 39: 331-342, 2010.

3. Reid KM, Ramos-De la Medina A and Donohue JH: Diagnosis and surgical management of gallbladder cancer: A review. J Gastrointest Surg 11: 671-681, 2007.

4. Hawkins WG, DeMatteo RP, Jarnagin WR, Ben-Porat L, Blumgart LH and Fong Y: Jaundice predicts advanced disease and early mortality in patients with gallbladder cancer. Ann Surg Oncol 11: 310-315, 2004.

5. Ootani T, Shirai Y, Tsukada K and Muto T: Relationship between gallbladder carcinoma and the segmental type of adenomyomatosis of the gallbladder. Cancer 69: 2647-2652, 1992.

6. Roa JC, Tapia O, Cakir A, Basturk O, Dursun N, Akdemir D, Saka B, Losada H, Bagci P and Adsay NV: Squamous cell and adenosquamous carcinomas of the gallbladder: Clinicopathological analysis of 34 cases identified in 606 carcinomas. Mod Pathol 24: 1069-1078, 2011.

7. Park SB, Kim YH, Rho HL, Chae GB and Hong SK: Primary carcinosarcoma of the gallbladder. J Korean Surg Soc 82: 54-58, 2012.
8. Liu DC and Yang ZL: MTDH and EphA7 are markers for metastasis and poor prognosis of gallbladder adenocarcinoma. Diagn Cytopathol 41: 199-205, 2013.

9. Giaginis C, Vgenopoulou S, Vielh P and Theocharis S: MCM proteins as diagnostic and prognostic tumor markers in the clinical setting. Histol Histopathol 25: 351-370, 2010.

10. MacCallum DE and Hall PA: The biochemical characterization of the DNA binding activity of pKi67. J Pathol 191: 286-298, 2000.

11. Mehrotra P, Gonzalez MA, Johnson SJ, Coleman N, Wilson JA, Davies BR and Lennard TW: Mcm-2 and Ki-67 have limited potential in preoperative diagnosis of thyroid malignancy. Laryngoscope 116: 1434-1438, 2006.

12. Brown DC and Gatter KC: Ki67 protein: The immaculate deception? Histopathology 40: 2-11, 2002.

13. Toschi L and Bravo R: Changes in cyclin/proliferating cell nuclear antigen distribution during DNA repair synthesis. J Cell Biol 107: 1623-1628, 1988.

14. Tachibana KE, Gonzalez MA and Coleman $\mathrm{N}$ : Cell-cycle-dependent regulation of DNA replication and its relevance to cancer pathology. J Pathol 205: 123-129, 2005.

15. Stoeber K, Tlsty TD, Happerfield L, Thomas GA, Romanov S, Bobrow L, Williams ED and Williams GH: DNA replication licensing and human cell proliferation. J Cell Sci 114: 2027-2041, 2001.

16. Madine MA, Swietlik M, Pelizon C, Romanowski P, Mills AD and Laskey RA: The roles of the MCM, ORC, and Cdc6 proteins in determining the replication competence of chromatin in quiescent cells. J Struct Biol 129: 198-210, 2000.

17. Chatrath P, Scott IS, Morris LS, Davies RJ, Rushbrook SM, Bird K, Vowler SL, Grant JW, Saeed IT, Howard D, et al: Aberrant expression of minichromosome maintenance protein-2 and Ki67 in laryngeal squamous epithelial lesions. Br J Cancer 89: 1048-1054, 2003

18. Davies RJ, Freeman A, Morris LS, Bingham S, Dilworth S, Scott I, Laskey RA, Miller R and Coleman N: Analysis of minichromosome maintenance proteins as a novel method for detection of colorectal cancer in stool. Lancet 359: 1917-1919, 2002.

19. Sirieix PS, O'Donovan M, Brown J, Save V, Coleman N and Fitzgerald RC: Surface expression of minichromosome maintenance proteins provides a novel method for detecting patients at risk for developing adenocarcinoma in Barrett's esophagus. Clin Cancer Res 9: 2560-2566, 2003.

20. Zhao J, Zhang X, Shi M, Xu H, Jin J, Ni H, Yang S, Dai J, Wu M and Guo Y: TIP30 inhibits growth of HCC cell lines and inhibits HCC xenografts in mice in combination with 5-FU. Hepatology 44: 205-215, 2006.

21. Shtivelman E: A link between metastasis and resistance to apoptosis of variant small cell lung carcinoma. Oncogene 14: 2167-2673, 1997.

22. NicAmhlaoibh R and Shtivelman E: Metastasis suppressor CC3 inhibits angiogenic properties of tumor cells in vitro. Oncogene 20: 270-275, 2001.

23. Xiao H, Palhan V, Yang Y and Roeder RG: TIP30 has an intrinsic kinase activity required for up-regulation of a subset of apoptotic genes. EMBO J 19: 956-963, 2000.

24. Li X, Zhang Y, Cao S, Chen X, Lu Y, Jin H, Sun S, Chen B, Liu J, Ding J, et al: Reduction of TIP30 correlates with poor prognosis of gastric cancer patients and its restoration drastically inhibits tumor growth and metastasis. Int J Cancer 124: 713-721, 2009.

25. Ito M, Jiang C, Krumm K, Zhang X, Pecha J, Zhao J, Guo Y, Roeder RG and Xiao H: TIP30 deficiency increases susceptibility to tumorigenesis. Cancer Res 63: 8763-8767, 2003.

26. Nishihara K, Nagai E, Izumi Y, Yamaguchi K and Tsuneyoshi M: Adenosquamous carcinoma of the gallbladder: A clinicopathological, immunohistochemical and flow-cytometric study of twenty cases. Jpn J Cancer Res 85: 389-399, 1994.

27. Kondo M, Dono K, Sakon M, Shimizu J, Nagano H, Nakamori S, Umeshita K, Wakasa K and Monden M: Adenosquamous carcinoma of the gallbladder. Hepatogastroenterology 49: 1230-1234, 2002.

28. Tye BK: MCM proteins in DNA replication. Annu Rev Biochem 68: 649-686, 1999.

29. Lei M and Tye BK: Initiating DNA synthesis from recruiting to activating the MCM complex. J Cell Sci 114: 1447-1454, 2001.

30. Zhang H, Zhang Y, Duan HO, Kirley SD, Lin SX, McDougal WS, $\mathrm{Xiao} \mathrm{H}$ and Wu CL: TIP30 is associated with progression and metastasis of prostate cancer. Int J Cancer 123: 810-816, 2008. 
31. Zhao J, Ni H, Ma Y, Dong L, Dai J, Zhao F, Yan X, Lu B, Xu H and Guo Y: TIP30/cc3 expression in breast carcinoma: Relation to metastasis, clinicopathologic parameters, and P53 expression. Hum Pathol 38: 293-298, 2007.

32. Gakiopoulou H, Korkolopoulou P, Levidou G, Thymara I, Saetta A, Piperi C, Givalos N, Vassilopoulos I, Ventouri K, Tsenga A, et al: Minichromosome maintenance 2 and 5 in non-benign epithelial ovarian tumours: Relationship with cell cycle regulators and prognostic implications. Br J Cancer 97: 1124-1134, 2007.

33. Reena RM, Mastura M, Siti-Aishan AM, Munirah MA, Norlia A, Naqiyah I, Rohaizak M and Sharifah NA: Minichromosome maintenance 2 is a reliable proliferative marker in breast carcinoma. Ann Diagn Pathol 12: 340-343, 2008.
34. Giaqinis C, Georgiadou M, Dimakopoulou K, Tsourouflis G, Gatzidou E, Kouraklis $\mathrm{G}$ and Theocharis S: Clinical significance of MCM-2, MCM-5 expression in colon cancer: Association with clinicopathological parameters and tumor proliferative capacity. Dig Dis Sci 54: 282-291, 2009.

35. Xiao H, Tao Y, Greenblatt J and Roeder RG: A cofactor, TIP30, specifically enhances HIV-1 Tat-activated transcription. Proc Natl Acad Sci USA 95: 2146-2151, 1998.

36. Shtivelman E: A link between metastasis and resistance to apoptosis of variant small cell lung carcinoma. Oncogene 14: 2167-2173,1997. 